\title{
Tollbooths: Congestion or Unhindered?
}

\author{
Zheyu Zhao \\ China, Beijing, changping country \\ 18811300277@163.com
}

Keywords: Merging area; Toll station; Fuzzy comprehensive evaluation; Real time control

\begin{abstract}
Through vissim simulation, we found that if the shape of the merging region is an isosceles trapezoid, the length of the merging region and the length of the straight line after the toll station are longer, the performance is better, but the cost will be increased accordingly. We analyzed the vehicle behavior and congestion, and then we propose a real-time control model for the vehicle merging process. The central idea of this model is to control the density of traffic exiting the toll booths near the maximum toll allowed by the toll road after the toll gate, to ensure that those vehicle who has paid the fee will not lead to the congestion when they through the merger area into the toll road. When the control device detects the upstream traffic volume is greater than the subsequent toll road throughput, using traffic lights to control the vehicles from the toll station exports so that can reduce congestion. Through the simulation and evaluation model, we compared the performance of our design area with the performance of the paid square, the conclusion is that the design area is superior to the original toll station under the assumption.
\end{abstract}

\section{Introduction}

However, with the rapid development of modern economy, the continuous expansion of urban scale , the rapid growth of urban motor vehicle ownership and private car ownership, the road traffic system has become overwhelmed and the traffic congestion is becoming more and more serious. Which again to toll station congestion is most serious. By optimizing the design of the merge area of the toll station, it is possible to improve the congestion situation. Traffic congestion will waste a lot of time, reduce efficiency, but also affect people's quality of life. So the road traffic system needs to be updated and improved to meet the more heavy traffic load.

\section{Assumptions and Justification}

To simplify the problem and make it easier to model realistic traffic conditions, we make the following basic assumptions, each of which is reasonable.

- Our study was conducted on six outbound lanes corresponding to three combined lanes.

- We use congestion dissipation, cost, incident rate, and throughput to evaluate the performance of the model, ignoring other possible factors.

- Our main research: In the case of congestion, toll stations are all in the export of saturated work

- Our model ignores other factors such as the impact on traffic after a traffic accident.

\section{Symbols}

Table 1 Notations

\begin{tabular}{ccc}
\hline Symbls & Definition & Unit \\
\hline$x_{i}$ & Criteria layer & \\
\hline $\mathrm{B}$ & Feature vector & \\
\hline $\mathrm{U}$ & $\begin{array}{c}\text { A set of evaluation } \\
\text { indicators }\end{array}$ & $\mathrm{m}$ \\
\hline $\mathrm{L}_{b}$ & Toll Plaza transition length & \\
\hline
\end{tabular}




\begin{tabular}{ccc}
\hline $\mathrm{A}$ & $\begin{array}{c}\text { Straight after the length of } \\
\text { the toll station } \\
\text { The length of the merge } \\
\text { region }\end{array}$ & $\mathrm{m}$ \\
\hline $\mathrm{H}$ & $\begin{array}{c}\text { Lane width } \\
\text { area }\end{array}$ & $\mathrm{m}$ \\
\hline $\mathrm{S}$ & $\begin{array}{c}\text { Consolidate the area of the } \\
\text { area }\end{array}$ & $\mathrm{m}$ \\
\hline $\mathrm{C}$ & Fence length on both sides & $\$$ \\
\hline $\mathrm{W}$ & $\begin{array}{c}\text { The width of the road } \\
\text { changing from the entrance } \\
\text { to the main line }\end{array}$ & $\mathrm{m}$ \\
\hline $\mathrm{b}$ & \begin{tabular}{c} 
cost \\
\hline
\end{tabular}
\end{tabular}

\section{Modoel I: Fuzzy comprehensive evaluation model based on AHP}

The establishment of model I. For an analysis of the performance of a specific toll plaza design that has been implemented, we develop an evaluation model that includes the important factors: accident prevention, throughput (hourly vehicles passing through the square at one end of the outgoing traffic lane), and cost (land and roadworks). These three factors are used as evaluation indexes.

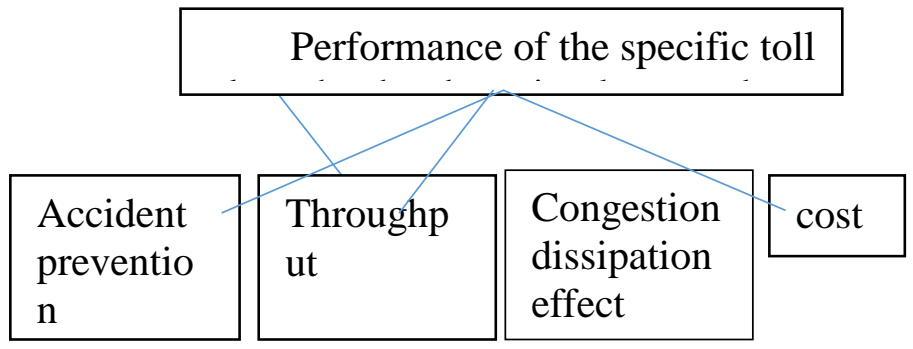

Fig. 1

Among them, the target layer: a specific toll square that has been implemented.

Criteria layer: accident prevention, throughput, congestion dissipation effect, cost.

Construct the judgment matrix. First, analyze the influence of the criterion layer $\left(x_{1}, x_{2}, x_{3}, x_{4}\right)$ on the target layer, and $p_{i j}$ is the influence ratio of $x_{i}$ and $x_{j}$ on the upper layer.

Table 2 the relative importance of $p_{i j}$ value

\begin{tabular}{c|c}
\hline Relatively important: $p_{i j}$ & definition \\
\hline 1 & $x_{i}$ is as important as $x_{j}$ \\
\hline 5 & $x_{i}$ is more important than $x_{j}$ \\
\hline 9 & $x_{i}$ is much more important than $x_{j}$ \\
\hline $1,1 / 5,1 / 9$ & $\begin{array}{c}\text { Corresponding to the above } \\
\text { relationship between the levels } x_{i} \text { and } \\
x_{j}\end{array}$ \\
\hline
\end{tabular}

After looking for information on the important relationship among accident prevention, congestion evacuation, throughput, and cost [1], we summarize the matrix M[1]: 


$$
M=\left(\begin{array}{cccc}
1 & 2 & 4 & 6 \\
1 / 2 & 1 & 2 & 4 \\
1 / 4 & 1 / 2 & 1 & 2 \\
1 / 6 & 1 / 4 & 1 / 2 & 1
\end{array}\right)
$$

Hierarchical Single Sequence and Its Consistency Test. Using MATLAB language to find the maximum eigenvalue of $\mathrm{M}: \lambda=4.0104$.Positive reciprocal matrix $\mathrm{M}$ consistency test, using T.L.Saaty consistency indicators:

$$
C I=(\lambda-n) /(n-1)
$$

According to Saaty's Stochastic Consistency Index Form:

\begin{tabular}{llllllllllll}
\hline n & \multicolumn{1}{c}{$\mathbf{1}$} & $\mathbf{2}$ & $\mathbf{3}$ & $\mathbf{4}$ & $\mathbf{5}$ & $\mathbf{6}$ & $\mathbf{7}$ & $\mathbf{8}$ & $\mathbf{9}$ & $\mathbf{1 0}$ & $\mathbf{1 1}$ \\
\hline RI & 0 & 0 & 0.58 & 0.90 & 1.12 & 1.24 & 1.32 & 1.41 & 1.45 & 1.49 & 1.51 \\
\hline \multicolumn{2}{c}{$\mathrm{RI}=0.90}$.
\end{tabular}

Consistency ratio $\mathrm{CR}=\mathrm{CI} / \mathrm{RI}=0.0104 / 0.90=0.0115$, i.e. by consistency test.

The weight vector is obtained by normalizing the difference between the indexes. For matrix eigenvectors with higher orders, if the matrices are the matrix that upper triangle is the inverse of the lower triangular, their eigenvalues and eigenvectors can be calculated by the following three simple approximation methods.

Power method:

Step1 take any n-dimensional normalized initial vector $w^{(0)}$.

Step2 calculates $\tilde{w}^{(k+1)}=A \tilde{w}^{(k)}, k=0,1,2, \cdots$.

Step3 normalized $\tilde{w}^{(k+1)}$, the expression is

$$
w^{(k+1)}=\frac{\tilde{w}^{(k+1)}}{\sum_{i=1}^{n} \tilde{w}^{(k+1)}} .
$$

Step4 for a given accuracy $\varepsilon$ in advance, when $\left|w_{i}^{(k+1)}-w_{i}^{(k)}\right|<\varepsilon, w^{(k+1)}$ is the required feature vector; otherwise return Step2.

Step5 Calculate the largest eigenvalue

$$
\lambda=\frac{1}{n} \sum_{i=1}^{n} \frac{\tilde{w}_{i}^{(k+1)}}{w_{i}^{(k)}}
$$

Sum method:

Step1 normalizes each column vector of A $\quad \tilde{w}_{i j}=\frac{p_{i j}}{\sum_{i=1}^{n} p_{i j}}$.

Step2 sum the $\tilde{w}_{i j}$ by line $\tilde{w}_{i}=\sum_{i=1}^{n} \tilde{w}_{i j}$.

Step3 normalizes $\tilde{w}_{i}$ to $w_{i}=\frac{\tilde{w}_{i}}{\sum_{i=1}^{n} \tilde{w}_{i}}, w=\left(w_{1}, w_{2}, \cdots, w_{n}\right)^{T}$.

Step4 Calculate $\lambda=\frac{1}{n} \sum_{i=1}^{n} \frac{(A w)_{i}}{w_{i}}$ as an approximation to the largest eigenvalue.

This method is actually making the A column vector normalized then averaging, as A's eigenvector. When $\mathrm{A}$ is a consistent matrix, each column vector is eigenvector, so if the inconsistency of A is not serious, it is reasonable to take the average value of A column vector (after normalization) as the approximate eigenvector.

Root method: 
Step1 normalizes each column vector of A $\tilde{w}_{i j}=\frac{p_{i j}}{\sum_{i=1}^{n} p_{i j}}$.

Step2 by the product of $\tilde{w}_{i j}$ row and the product of the n power, that is $\tilde{w}_{i}=\left(\prod_{j=1}^{n} \tilde{w}_{i j}\right)^{1 / n}$.

Step3 normalizeing $\tilde{w}_{i}, w_{i}=\frac{\tilde{w}_{i}}{\sum_{i=1}^{n} \tilde{w}_{i}}, w=\left(w_{1}, w_{2}, \cdots, w_{n}\right)^{T}$.

Step4 Computeing $\lambda=\frac{1}{n} \sum_{i=1}^{n} \frac{(A w)_{i}}{w_{i}}$ as an approximation to the largest eigenvalue.

Using one of these methods, the normalized feature vector (weight vector) is obtained by MATLAB: $\mathrm{B}=(0.3533,0.2451,0.2075,0.1941)$.

In the performance analysis, the weight of security prevention, throughput and cost are $(0.3533,0.2451,0.2075,0.1941)$ respectively.

According to the purpose to determine the evaluation index set: $U=\left\{u_{1}, u_{2}, u_{3}, u_{4}\right\}, u_{i}$ correspond to the above four indicators. Giving the evaluation level set: $V=\left\{v_{1}, v_{2}, \cdots, v_{n}\right\}$, where $v_{n}$ respectively said poor, ordinary, medium, good, excellent. Determine the weight of each evaluation index $w=\left\{\mu_{1}, \mu_{2}, \mu_{3}, \mu_{4}\right\}$, the weight reflects the evaluation index in the comprehensive evaluation of the importance of degree, and $\sum \mu_{i}=1$.

In order to improve the accuracy of the model, this model uses the previous analytical hierarchy model to obtain the weight (weight vector) $\mathrm{w}=(0.3533,0.2451,0.2075,0.1941)$.

$\{x(i, j), i=1 \cdots n, j=1 \cdots m\}$ is evaluation Indicator Sample Set Data of all the $\mathrm{m}$ schemes and $\mathrm{n}$ evaluation indicators, and each indicator value $x(i, j)$ is non-evaluation. In order to determine the fuzzy evaluation matrix of the relative membership of a single evaluation index, the sample data $\{x(i, j), i=1 \cdots n, j=1 \cdots m\}$ needs to be standardized. In order to maintain as much as possible the change of the evaluation index value of the information for the larger the better indicators of the standard treatment formula can be taken as:

$$
r(i, j)=x(i, j) *\left[x_{\max }(i)+x_{\min }(i)\right]
$$

For the smaller the better the type of standard processing formula can be taken as:

$$
r(i, j)=\left[x_{\max }(i)+x_{\min }(i)-x(i, j)\right] *\left[x_{\max }(i)+x_{\min }(i)\right]
$$

Where $x_{\min }(i) 、 x_{\max }(i) 、 x(i, j)$ are the minimum, maximum and intermediate optimum values of the ith index of the scheme set respectively. $r(i, j)$ is the standardized evaluation index value, that is the $\mathrm{i}$-th evaluation index of the $\mathrm{j}$-th scheme is subordinate to the relative membership value $i=1 \cdots n, j=1 \cdots m$. The fuzzy evaluation matrix $R=(r(i, j))_{n \times m}$ can be constructed by using these $r(i, j)$ values as elements. We will be divided into ten grades, the higher the level, the better, to avoid the result is too small, the change is not obvious.

The weight value of each evaluation index and the relative membership value $r(i, j)$ of the corresponding evaluation index of each scheme are multiplied and added and subtracted to obtain the comprehensive index value $z(j)$.

$$
z(j)=\sum_{i=1}^{n} w_{i} r(i, j)
$$

The bigger of the index $z(j)$, the better of the $j$-th scheme, so it can make scientific decision. In order to facilitate the calculation and comparison, we will have to implement the toll plaza simulation data all subjective as 5 , for our design of the regional simulation data we see it is a multiple of the 
implementation, and then divided into ten grades.

\section{On the Performance of the Toll Plaza has been analyzed}

Factor analysis. We use the dissipation effect, cost, accident rate, throughput to evaluate the performance of the model.

The queuing length and throughput of the merging area jamming dissipation effect are obtained by vissim simulation.

Definition of Congestion Dissipation: Let D be the queue length of the transition section of toll plaza before congestion dissipation, $\mathrm{Dp}$ is the queuing length of transition section of toll plaza after adopting control strategy. The congestion dissipation effect is defined as: $E_{p}=\frac{D-D_{p}}{D} \times 100 \%$

Table 3 Traffic Congestion Degree of Expressway Main line Toll Station[2]

\begin{tabular}{|c|c|c|c|}
\hline Degree of congestion & Mild congestion & Moderate congestion & Severe congestion \\
\hline Queue length & $(4 / 1 \mathrm{Lb} 2 / 1 \mathrm{Lb})$ & $(2 / 1 \mathrm{Lb}$ 4/3Lb) & $(4 / 3 \mathrm{Lb} \mathrm{Lb})$ \\
\hline
\end{tabular}

$\mathrm{L}_{b}$ is the length of the toll plaza transition section

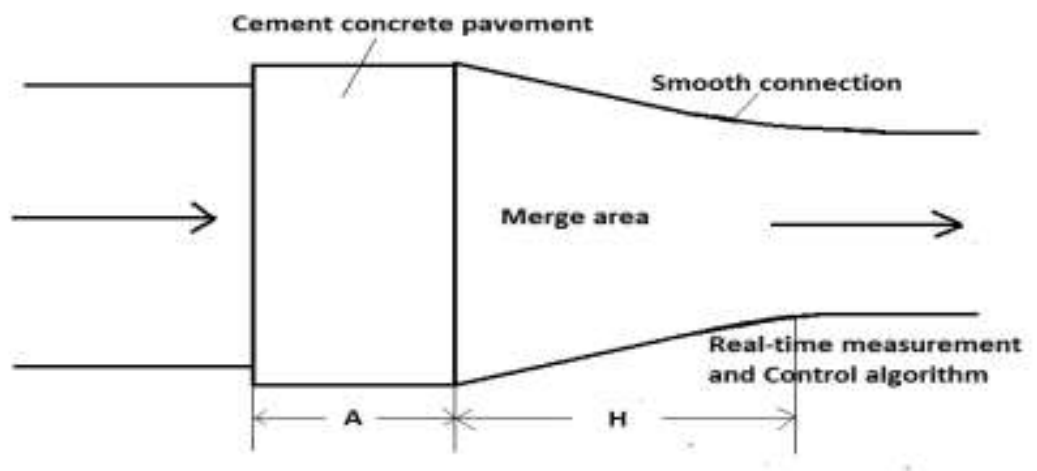

Fig.2

Consolidated Regional Cost Analysis. The geometric model of the area can be represented as follows:

The construction cost of the area is mainly proportional to the area and the length of the two sides of the fence, so only need to determine the shape and size of the premise, the fan-in area of the area and the surrounding length of the fence area can estimate the construction cost .

Lane lane and freeway lane width is equal and $\mathrm{h}$. Then the area:

$$
S=A^{*} L^{*} h+(B * h+L * h) * H / 2
$$

Fence length on both sides:

$$
C=2 * \alpha+2 * \sqrt{H^{2}+[(L-B) * h / 2]^{2}}
$$

Set the cost coefficient (including construction and land lease) of the ground $\alpha$, fence construction cost coefficient $\beta$. So the cost of the final area can be estimated as: $W=\alpha^{*} S+\beta^{*} C$ 
Accident rate Analysis. In the merged zone entrance and exit section, it is necessary to guide the vehicle to enter and exit the area smoothly and smoothly. The gradual change rate requirement is gentle and easy for the vehicle to drive. Otherwise it is easy make the driver a sense of displacement, resulting in improper operation and endanger road safety. The merging area gradient rate " $\mathrm{l}$ " is the ratio of the road width (b) and the length of the transition zone $(\mathrm{H})$, which is added to the main line section, ie $1=\mathrm{b} / \mathrm{H}$. According to the experience of the United States and Japan, the vehicle from a straight line into the other lane of the skew ratio of about 0.9 , the driver is generally no driving sense of displacement, no discomfort.

Accordingly, when the vehicle travels at speeds of $40 \mathrm{~km} / \mathrm{h}$ and $60 \mathrm{~km} / \mathrm{h}$, the length of the transition section should be $40 \mathrm{~m}$ and $55 \mathrm{~m}$, with a gradient of $1 / 15$ and $1 / 18$. The current number of toll lanes is usually determined by traffic capacity and predicted traffic volume, generally more than the main lane 1-3, the length of the transition section of $25 \mathrm{~m}$ or so. Gradual rate can only be 1/5 1/ 7 between the values, significantly lower than the experience value.

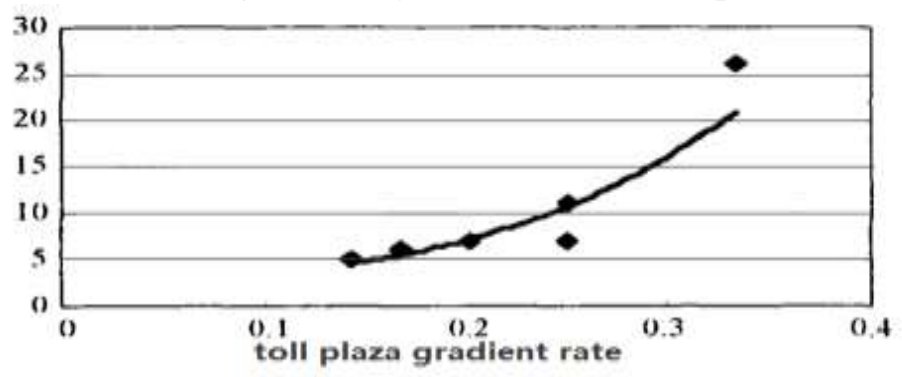

Fig.3

Fig.3shows the relationship between the gradual change rate of highway and the traffic accident. From the figure, we can see that with the increasing rate of regional gradient, the number of traffic accidents will increase and the security of the area will decrease. Through the data regression analysis, the correlation model between the regional gradient and the traffic accident number was obtained:

$Y=1.4231 e^{8.0664 x}$

In the formula, $\mathrm{Y}$ - the regional gradient of the traffic accident rate;

$\mathrm{X}$ - the regional gradient rate.

From this model we can see that the frequency of traffic accidents and regional gradient is proportional to. If the gradient length is insufficient and the gradient rate is too large, it will cause the vehicle safety to judge the sight line insufficiency, and can not meet the safety requirement of entering the area vend lead to the traffic accident.

\section{Performance Analysis of Specific Toll Plazas that have been Implemented.}

According to the survey, etc in the toll station accounted for about $80 \%$. As shown in figure (4).New Jersey daily trafficAs shown in figure (5) Capacity of a toll lane in various US toll systems, As shown in Table 4.

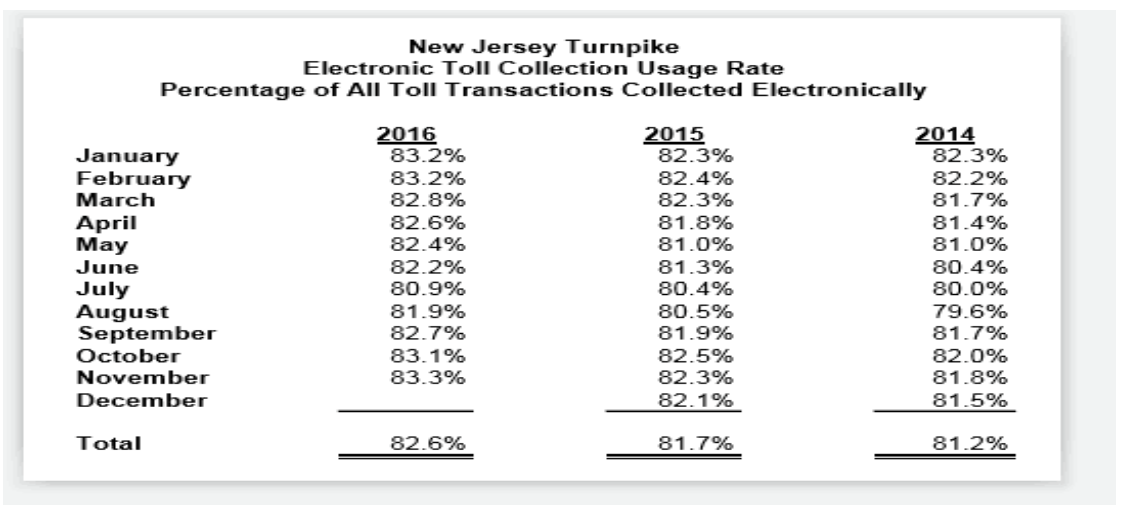

Fig.4 


\begin{tabular}{|c|c|c|c|c|c|c|c|c|c|}
\hline Start Time & End Time & Hour* & $\begin{array}{c}\text { Influx } \\
\text { (cars/min) }\end{array}$ & $\begin{array}{c}\text { Fonrier Approx } \\
\text { of Influxx** }\end{array}$ & Start Time & End Time & Hom ${ }^{*}$ & $\begin{array}{c}\text { Inffux } \\
\text { (cars/min) }\end{array}$ & $\begin{array}{c}\text { Fourier Approx } \\
\text { of Influx }\end{array}$ \\
\hline $12: 00 \mathrm{AM}$ & $1: 00 \mathrm{AM}$ & 0.5 & 15.44 & 15.16272478 & $12: 00 \mathrm{PM}$ & $1000 \mathrm{PMI}$ & 12.5 & 41.72 & 41.63346483 \\
\hline $1: 00 \mathrm{AM}$ & $2: 00 \mathrm{AM}$ & 1.5 & 15.32 & 15.42467822 & $1100 \mathrm{PM}$ & 2:00 PM & 13.5 & 44.54 & 44.44085865 \\
\hline $2: 00 \mathrm{AM}$ & $3: 00$ AM & 2.5 & 15.16 & 15.18796896 & $200 \mathrm{PM}$ & $300 \mathrm{PM}$ & 14.5 & 48.88 & 49.29448007 \\
\hline $3: 00 \mathrm{AMI}$ & $4: 00 \mathrm{AM}$ & 3.5 & 19.90 & 19.81853474 & $3: 00 \mathrm{PMI}$ & 4:00 PM & 15.5 & 53.20 & 52.55619485 \\
\hline $4: 00 \mathrm{AM}$ & $5: 00 \mathrm{AM}$ & 4.5 & 47.09 & 47.22251986 & 4.00 PM & 500 PM & 16.5 & 51.61 & 52.21058951 \\
\hline $5: 00 \mathrm{AM}$ & $6: 00 \mathrm{AM}$ & 5.5 & 89.95 & 89.61825869 & 500 PM & $6000 \mathrm{PM}$ & 17.5 & 48.38 & 48.16410937 \\
\hline $6: 00 \mathrm{AM}$ & $7: 00 \mathrm{AM}$ & 6.5 & 105.9 & 106.4828683 & $6.00 \mathrm{PM}$ & $700 \mathrm{PM}$ & 18.6 & 39.72 & 39.50374966 \\
\hline $7: 00 \mathrm{AM}$ & $8: 00$ AM & 7.5 & 85.52 & 84.72959878 & $7: 00 \mathrm{PMI}$ & 8:00 PMI & 19.5 & 30.51 & 31.11397219 \\
\hline $8: 00 \mathrm{AM}$ & $9: 00 \mathrm{AM}$ & 8.5 & 54.68 & 55.57942216 & $8000 \mathrm{PM}$ & $900 \mathrm{PMI}$ & 20.5 & 29.48 & 28.86864636 \\
\hline $9: 00 \mathrm{AM}$ & $10: 00 \mathrm{AM}$ & 9.5 & 43.11 & 42.42662327 & $9000 \mathrm{PM}$ & $10,00 \mathrm{PM}$ & 21.5 & 26.82 & 27.19686700 \\
\hline $10: 00 \mathrm{AM}$ & $11: 00 \mathrm{AM}$ & 10.5 & 40.16 & 40.49538486 & $10: 00 \mathrm{PM}$ & $1100 \mathrm{PM}$ & 22.5 & 21.21 & 21.26085220 \\
\hline $11: 00 \mathrm{AM}$ & $12-00 \mathrm{PM}$ & 11.5 & 40.85 & 40.83544106 & $11500 \mathrm{PMI}$ & $12: 90 \mathrm{AM}$ & 23.5 & 17.22 & 16.91795178 \\
\hline
\end{tabular}

Fig.5

Table 4 Capacity of a toll lane in various US toll systems[4]

\begin{tabular}{cc}
\hline Typesof charges & $\begin{array}{c}\text { The capacity of each toll } \\
\text { channel(pculh) }\end{array}$ \\
\hline $\begin{array}{c}\text { conventional (human-staffed) } \\
\text { tollbooths } \\
\text { exact-change (automated) } \\
\text { tollbooths }\end{array}$ & 350 \\
electronic toll collection booths & 650 \\
\hline
\end{tabular}

Therefore, we set that one of the six tolls is the conventional (human-staffed) tollbooths, one for the exact-change (automated) tollbooths and four for the electronic toll collection booths.

The composition of traffic flow. As shown in Fig.6 Traffic flow settings, 6000(pcu/h) As shown in Fig.6.

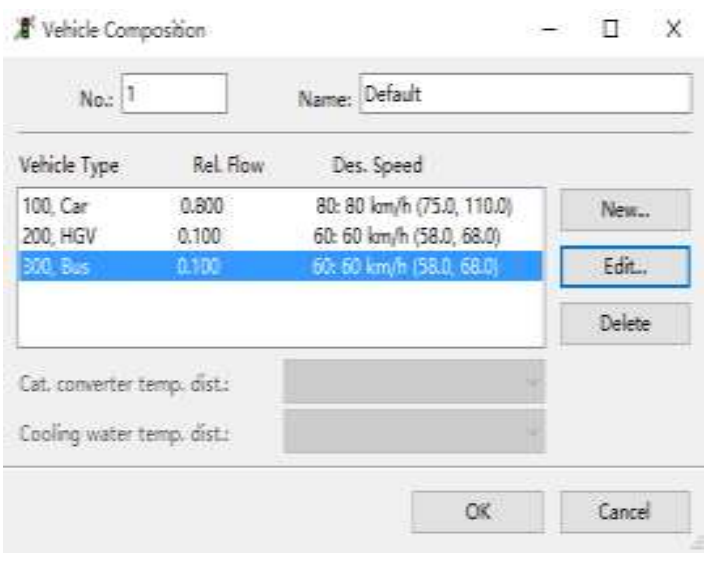

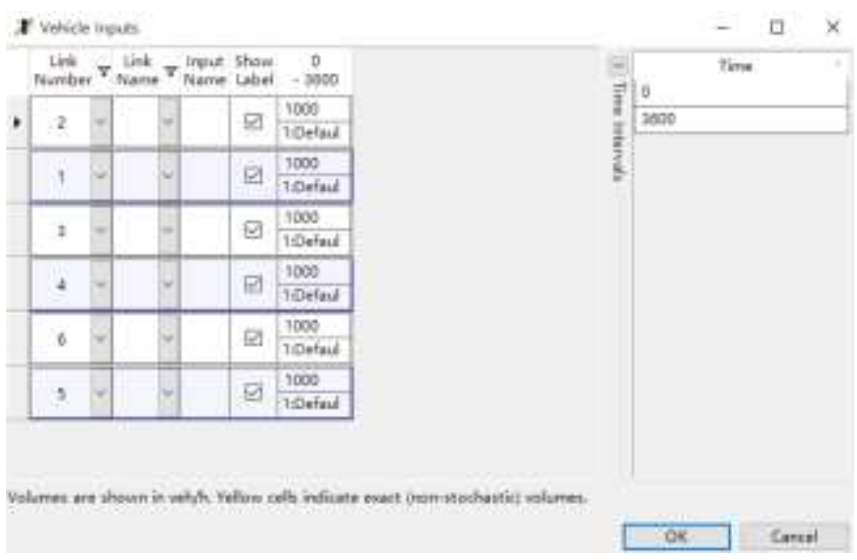

Fig.6

Simulation of existing toll booths. Simulation Fig.7

Results: In the case of relatively large traffic flow in the merger area is more serious congestion.

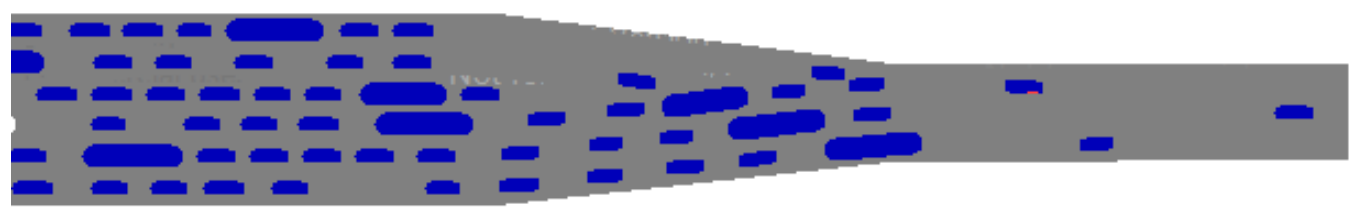

Fig.7 
Cost: $\quad A=80, H=126, B=6, L=3, h=3.5$

Incident rate: $\mathrm{A}=80, \mathrm{H}=126$

Congestion evacuation effect: $\quad \mathrm{D}=1 / 2 * \mathrm{H}$

Conclusion. Using the model-specific algorithm, we come to the integrated index:

$\mathrm{z}(1)=0.3533 * 5+0.2451 * 5+0.2075 * 5-0.1941 * 5=3.059$.

\section{Model II: Simulation Design Model}

The Design of the Area Shape. Based on the shape of all toll stations at home and abroad are isosceles trapezoid, so we decided to continue to follow the isosceles trapezoidal shape.

The Design of the Area size. 1 Through the method of control variable and the method of vissim simulation, we determine the relationship between throughput and the merger area.

2 to study the relationship between throughput and the size of the merger area.

The size of the merge region is determined mainly by the length A of the toll road and the height $\mathrm{H}$

of the merged region trapezoid, where the merged region is assumed to be an isosceles trapezoid. As shown in Fig8.

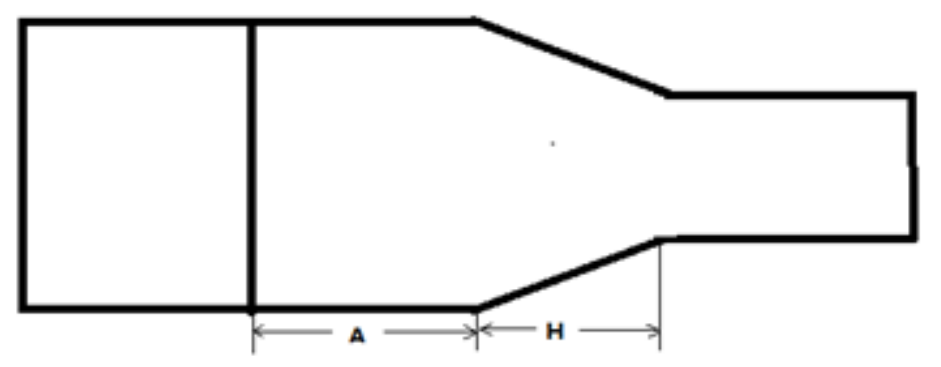

Fig8

Based on the 2005 MCM B problem, we assume that the number of lanes and combined lanes of toll stations are 6 and 3, respectively, so the trapezoidal top and bottom of the merged region unchanged.

By the control variable method, the merging mode remains the same as the merging mode in real life, that is random merging.

(1) Keeping $\mathrm{H}$ unchanged, changing $\mathrm{A}$, through vissim simulation, increase $\mathrm{A}$ and change throughput to 2952 ( pcu / h), reduce A and change throughput to 1872 (pcu / h), the original throughput of 2052 (pcu / H). We found that the larger the A, the greater the throughput. The simulation results are shown in Fig9.

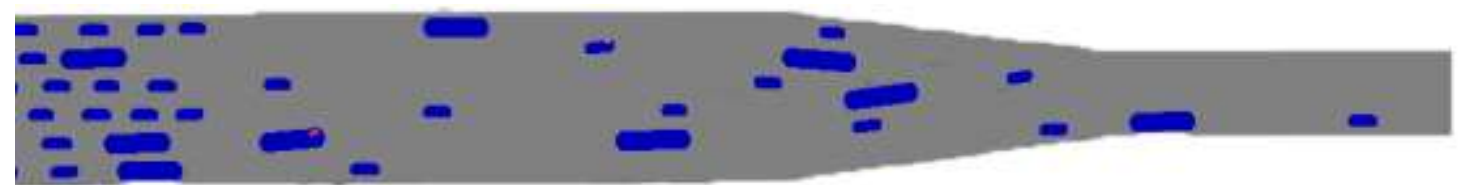

Increase A

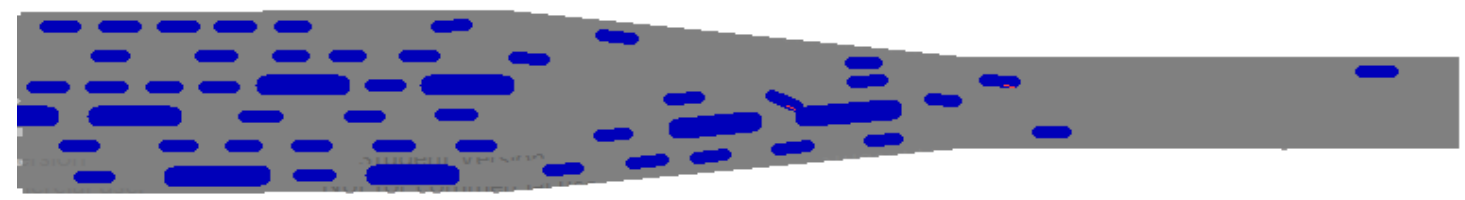

Reduce A

Fig9

(2) Now the merge mode and $A$ is maintained, changing $H$, increasing the $H$, the throughput becomes 2700 (pcu / h) by vissim simulation, and reducing the $\mathrm{H}$, the throughput becomes 1800 (puc 
/ h). We found that the larger the $\mathrm{H}$, the greater the throughput. Simulation results shown in Fig10.

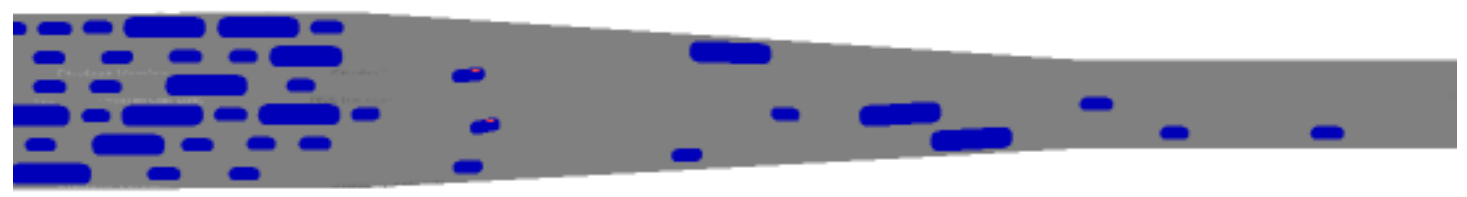

Increase $\mathrm{H}$

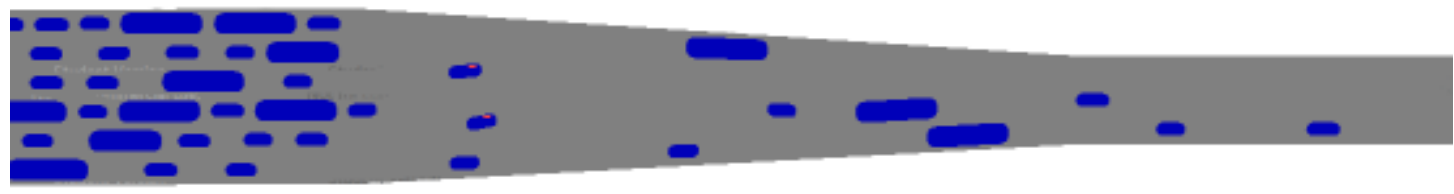

Increase H

Fig10

The Design of Merge Mode. We found by field investigation: the existing toll station export areas mainly exist the following areas can be improved.

The design ground for the special floor of the toll station outbound area (with wear-resistant cement concrete pavement)is not long enough.

Vehicles in the square frequent braking and start asphalt concrete pavement will cause serious rut and the phenomenon of the passage, endangering the safety of the square and affect the normal use. In addition, the temporary parking vehicles in the channel often accompanied by serious oil spills. Especially diesel vehicles oil spill will lead to rapid damage to the asphalt pavement. Therefore, from the square of the durability, easy to clean and so on, the toll plaza must use concrete pavement. Opening of the toll plaza is widespread pavement length of concrete phenomenon, will lead to the accident, which is more obvious in the main line of toll plaza. According to the survey data show that the cause of this phenomenon is due to the vehicle just out of the toll station, vehicle start and eager to change lane and brake start again.

The toll road to the design of high-speed road section.

Due to the charge out of the fan-in area design is small, in the peak period, once the larger size of the vehicle is more likely to cause congestion, and should not be diverted.

Merge mode is single

Vehicles are free to merge after leaving the toll island. Resulting in large vehicles and small vehicles interspersed with, due to vehicle performance and driver driving habits and other reasons, prone to traffic accidents.

Conclusion: In view of the above problems how to change the toll station exit area size, size and merge mode to improve the performance of the toll station, we made the following analysis to clarify the direction of improvement of our model.

Congestion analysis. The arriving traffic typically enters the toll station area and eventually approaches the merge area on the total number of lanes of the vehicle. The arriving traffic originates from the upstream transport infrastructure. If the capacity of the upstream road is equal to or less than the capacity of the downstream infrastructure, the vehicle queuing time and the delay of the vehicle queuing can be minimized. If the toll station can provide a high enough service capacity, the vehicle queuing time and the delay of the vehicle queuing can be minimized. However, if the upstream infrastructure capacity is higher than the downstream capacity, regardless of the service capacity of toll stations, car queuing is inevitable.

After combining from B to L lanes, the vehicle enters an exit highway with $L$ lanes and a corresponding flow capacity. It is not necessary to incorporate flow control, if the toll station has a total outflow (occasionally) higher than the outflow. The introduction of electronic charging technology to increase the throughput of the toll station, so more and more charging square in the future may face merger control issues.

Vehicles that leave the toll station from a total of B lanes change the lane appropriately (usually trapezoidal) within the merging zone in order to be able to properly enter the exit of the L lanes. The merging process can be quite complex in terms of required vehicle maneuvering, and the process of 
simulating vehicle maneuvering merges can be a challenge for simulation software. In fact, lane change components are a weakness of simulation software, especially when applied to special merged transport infrastructures as toll plazas.

Fortunately, the complexity of microscopic vehicle merging behavior is not reflected in the real-time merged traffic control actions envisioned here, since the underlying assumption that real-time traffic control merges is macroscopical and is related to the notion of a basic map well known to the public. The figure(11) shows a typical flow-density plot for the merged area, where the flow is the exit flow and $\mathrm{N}$ is the number of cars included in the consolidation zone. When $\mathrm{N}$ hours, the merger conflict is scarce and easy to solve, while the outlet flow is correspondingly low. As $\mathrm{N}$ increases, the merge conflict may increase, but also increases until a certain value, the outlet flow reaches the downstream capacity. If $\mathrm{N}$ exceeds, the merging conflict becomes more frequent and severe, causing a large number of vehicles to decelerate and reaccelerate, which reduces the exit flow to a lower value .

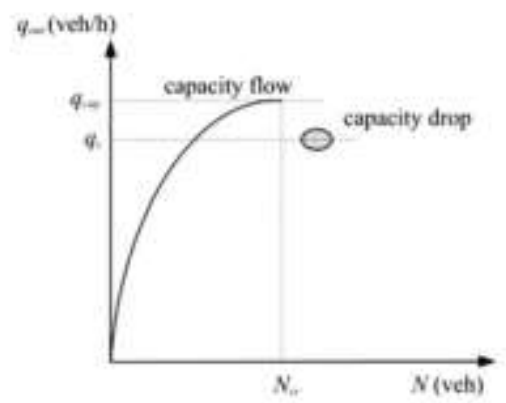

Fig.11

The phenomenon of decline in capacity has been repeatedly observed and reported, with most of the content coming from the highway merging area. It is observed that the traffic flow in the merged area can be increased to a capacity value at a relatively high average speed. If the total arriving traffic continues to increase beyond this value, the traffic accident is most likely to occur, and a fall in speed and more traffic will follow. The flow downstream of the congested source being measured is found to be about 5-20\% lower in different studies than .

Actual data analysis from the highway ramp merging area shows that even under similar environmental conditions, traffic accidents can still occur on different days and with different flow values q_cap. Naturally, in the case of unfavorable environmental conditions, the difference in flow capacity becomes more pronounced. In contrast, the critical occupancy of the capacity flow is found to be fairly constant, even under adverse environmental conditions. The results of these studies suggest the introduction of a control scheme that takes action on arriving traffic flows in order to maintain. If necessary, the number of vehicles in the merging area $\mathrm{N}$ close to its critical value Ncr such that the outlet maximizes the flow, Infrastructure efficiency[5].

Vehicle Behavior Analysis. The characteristics of the traffic flow at the toll station are complex, and the main characteristics of the vehicle behavior can be analyzed from the following aspects:

1) Toll lane setting characteristics

As the vehicle before entering the toll station in accordance with the arrangement of the toll lane will be separate models, resulting in the outbound large vehicles near the road on both sides. Due to the slow start of large vehicles and the large space occupied by the line, most of the large vehicles have a waiting process into the middle lane.

2) the impact of vehicle interludes

Toll Plaza, due to vehicle acceleration is slow and slow start, the vehicle will choose to change the different lanes and overtaking, reaching the fastest high-speed results. Vehicle interludes, the toll plaza within the traffic situation will have a great impact. In addition to the impact will be interspersed with the normal driving lane vehicles, but also prone to traffic accidents.

3 ) the vehicle speed distribution characteristics of the toll plaza

The speedup process of each vehicle is different in the process of speeding off the entire fan-in area 
after the vehicle leaves the toll island, which is determined by the vehicle's own performance and the driver's own habits. Therefore, the distribution of the vehicle speed in this region is discrete, and it is not possible for a vehicle traveling out of the toll island to enter the expressway at the same time.

4) Vehicle queuing characteristics

When the front section of a road accident and other emergencies led to the fan into the area of congestion, the vehicle is fan-shaped distribution in the fan-in area and the highway connection mouth, waiting in line, followed by.

5) the driver's psychological characteristics

If the combined regional design is too large, the driver to see a wide path to relax and vigilance, speed will continue to increase, but likely to cause more serious traffic accidents.

Summary: toll station vehicles will be separated according to the size of the vehicle, in order to prevent the size of vehicles interspersed with the block can be designed for large vehicles, roads, guide carts directly into the expressway slow lane, rather than occupying small vehicles lane. When a slight congestion or even a heavy congestion, due to the queuing characteristics of the vehicle, the end of the merged area should be connected with the highway to smooth connection to facilitate the turning into the vehicle[6].

Regional Model of Toll Station Exit. Based on the above analysis, we present a new toll station model which can theoretically improve the performance of the existing toll station model.

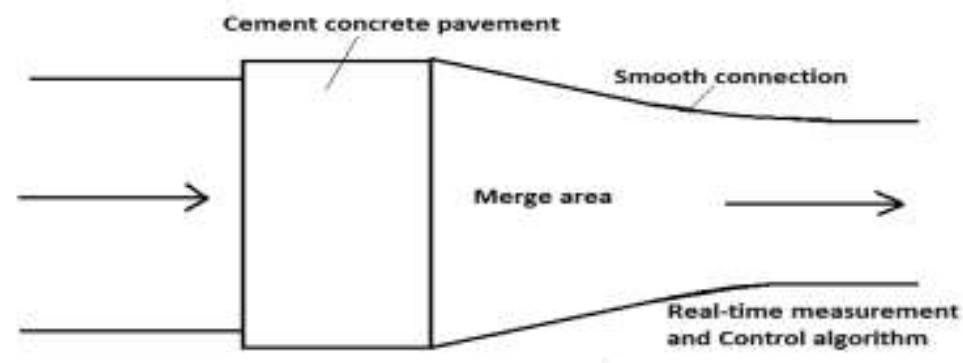

Fig12

Smooth connection: change the part which is connecting with the top of trapezoidal and highway to the form of smooth conection.

Real-time measurement and Control algorithm: A combined mode controlled by traffic lights to improve congestion and throughput.

Now,we are introducing a new model of merge mode.

Control device. The control device is a traffic light located downstream of the toll station, which is located just upstream of the merge area. Traffic lights can be applied separately to individual lanes or to lane groups. The control means may be mounted on only a portion of the entry lane. Because toll stations need to set up some green channel for special vehicles. For these vehicles, they should not be restricted even when the congestion.[7]

Real-Time Measurement or Estimation. In order to keep the number of vehicles (N) close (Ncr), the feedback control section requires a value that can be measured or estimated in real time. This amount can be measured directly using a video sensor.

Another possibility is to treat occupancy (o) as a controlled variable. There is a problem of how to find the exact location of the measurement occupancy. The measuring device for occupancy should preferably be placed where the vehicle decelerates severely and is congested or congested just before the front end. Because once congestion occurs, it will spread along the road upstream. Therefore, the feedback control algorithm of the measuring device should be placed in:

- Do not place the device downstream of congestion, otherwise congestion is not visible, and control objectives may not be achieved.

- Keep as close as possible to the upstream of the position so that the delay in triggering the control reaction is as small as possible. 
In general, the functional area for control may have to extend downstream to cover the location where congestion occurs. In general, the most congested locations are most likely to be on the downstream freeways. [8]

Control Algorithm. The control algorithm receives the real-time measurements or estimates of $\mathrm{N}$ and drives the control devices in order to maintain $\mathrm{N}=\mathrm{N} \_\mathrm{cr}$, which maximizes the merge area exit flow. This is the main goal of the control algorithm

Feedback control is the most important part of the control algorithm. At the end of each count cycle, this cycle of traffic data is saved for generating the next count cycle of traffic, whose purpose is to keep N=N_crin the area. We design the simulation model by using the ALINEA algorithm[10] the formula of this algorithms is :

$$
q(k)=q(k-1)+K_{R}[\hat{o}-o(k-1)]
$$

where $\mathrm{k}=1,2, \ldots=$ discrete time index; $\mathrm{q}(\mathrm{k})=$ controlled enteringflow (veh/h) to be implemented during the new period $\mathrm{k} ; \mathrm{o}(\mathrm{k}-1)=$ last measured occupancy $(\%)$ averaged over all exit lanes; $K_{R}>0$ =regulator parameter; and $\hat{o}=$ set (desired) value for the occupancy which may be set equal to $o_{c r}$ for maximum exit flow.

The traffic flow $\mathrm{q}(\mathrm{k})$ should be limited in a reasonable range. Once this value in the process of simulation go beyond this range, the simulation program should be immediately stopped and put the last reasonable value of $\mathrm{q}(\mathrm{k}-1)$ as the initial value in the next counting cycle. Of course, this range should also be broadened appropriately in order to make process more simple, which will not interrupt at the peak of traffic flow and show "errors" frequently.

After several simulation practice,we found that the reasonable values, as a trade-off of the mentioned aspects, are $q_{\max } \approx 1.2 q_{\text {cap }}$ and $q_{\text {min }}=0.5 q_{\text {cap }}$.

Simulation process. Based on the simulation of vissim software, the size of our new model described above is input into the model. The maximum throughput of the toll road in the downstream (three lanes) is $\approx 2900 \mathrm{veh} / \mathrm{h}$. Initially, the upstream capacity is designed to $1000 \mathrm{veh} / \mathrm{h}$.In the first 10 minutes, the traffic flow will be increased to 4000veh / h linearly. In the next 20 to 30 minutes , the traffic flow from upstream evenly is reduced to 1000veh / $\mathrm{H}$ and finally zero.

The regulator is activated at each $30 \mathrm{~s}$. The flow value $\mathrm{q}$ is determined by equation (1). In practice, the traffic lights are controlled by each lane. Each traffic signal has a constant green phase of $4 \mathrm{~s}$, during which approximately two vehicles are allowed to pass. The signal period c (s) consists of a constant green phase and a variable red phase. Therefore, the implementation of the flow q requires equal flow periods for all channels, which satisfy.

$$
\mathrm{q}=(2 * M / c) * 3600 \Rightarrow c=7200 * M / q
$$

To avoid the possibility that the green or red light at the same time, all lanes are divided into two groups, $\{1,3,5\},\{2,4,6\}$. The start of the cycle between two groups is set to a small delay.

Since the randomness of vissim simulation software is large, the same data under different simulation conditions may produce different results. For this problem, the solution is to run multiple simulations for each simulation scenario, taking the average, and doing 10 repeats for each case. The evaluation criteria used were Average Vehicle Delay Time (AVD) (s / veh / km)

\section{Simulation Results}

No control case 


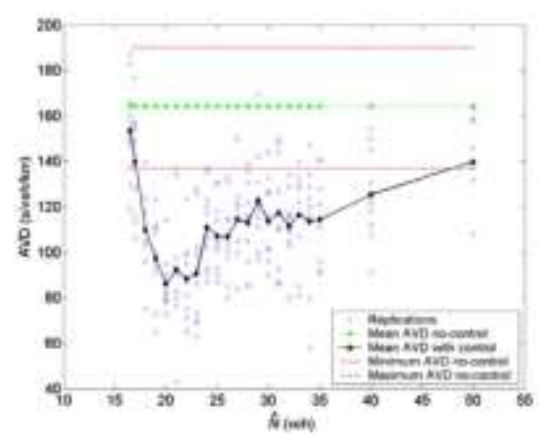

Fig. 13

When the control equipment is not erected, if the traffic volume in the process of simulation is lower than the export capacity, the vehicle will merge into the toll road in the merging area and enter the subsequent toll road at a faster speed. When the number of cars approaches, you can see the serious conflict and deciline of speed rapidly, which will lead to the congestion in the merger area near the exit [9].The congestion gradually lengthened until the influx of vehicles toll station normal entry . The average AVD was 164 by simulation.(fig.13)

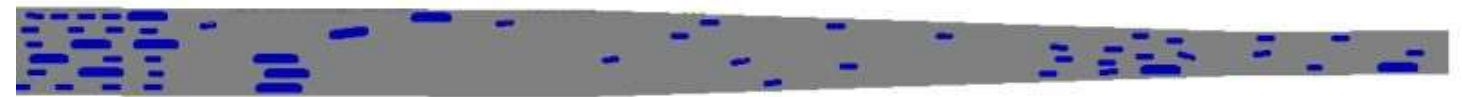

Fig.14

Fig.14 shows the curve model for the relationship between the throughput q_outand the number of vehicles $\mathrm{N}$ for a particular simulation process. It can be seen that the throughput q_out is increasing close to the throughput upper limit q_out, and after 10 minutes the congestion area , The throughput is reduced to around 2400 with the decrease in the vehicle into the first increase after a slight decrease with the vehicle into the reduced to zero.

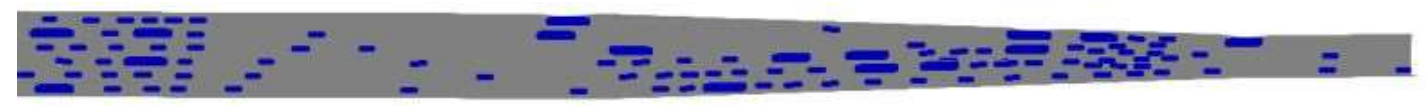

Fig. 15

Fig.15 shows the congestion situation in the consolidation area when the number of vehicles entering the vehicle exceeds the upper limit of downstream throughput.

Control case. When the number of vehicles entering is lower than the upper limit of throughput, the simulation results are almost the same as those without control. When the traffic volume is close to the upper limit of throughput, the controller starts to work, and the exit of the toll station can be seen alternately from red to green. The volume of vehicles released significantly slowed down, which traffic flow is controlled to the upper limit of throughput. we can see that the toll station upstream don't format congestion. Observeing the relationship between the throughput q_out and the number of vehicles $\mathrm{N}$ at this time, it can be seen that when the throughput reaches the upper limit, it does not decrease but keeps flooding around the limit value. It is until the end of the simulation that the value decreas to zeor . The average AVD is 130 or so(fig.16).
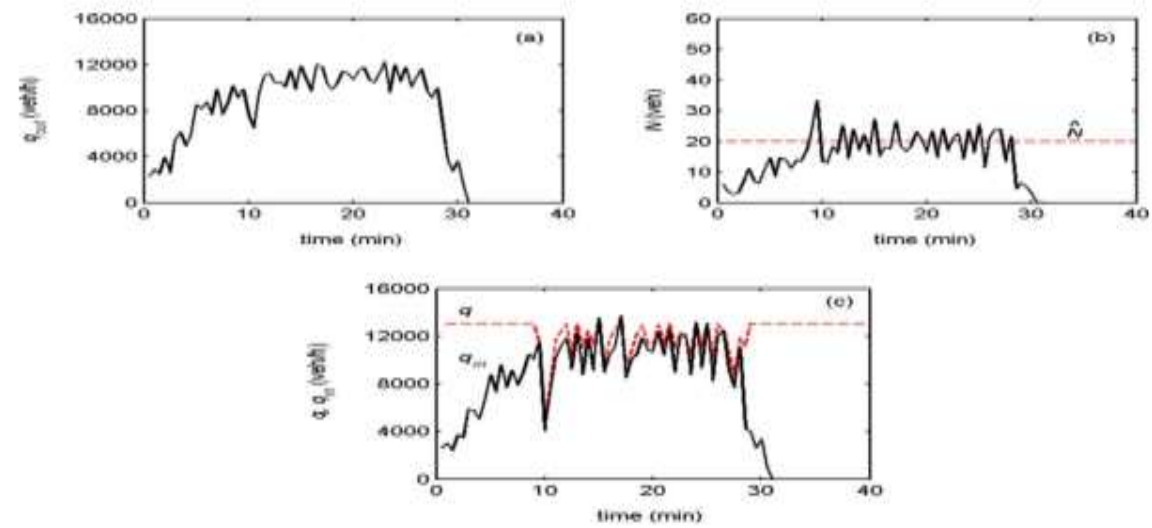

Fig.16 


\section{Conclusion}

The comprehensive evaluation value of the combined regional performance is $\mathrm{z}(2)=0.3533 * 7+0.2451 * 6+0.2075 * 6-0.1941 * 8=3.4322>3.059$.

When a heavier traffic arrives, a new control mode can be used to mitigate congestion in the merged zone at the toll exit and the throughput maintain a large number above. There are not too many vehicles queuing up into the toll booth as well. The simulation results show that the vehicle average delay time (AVD) is decreased by 12.2 percent regarding of no control case.Thus,new traffic control system is good gor improving the property of toll station.

\section{Model Analysis and Sensitivity Analysis}

\section{Performance of Models with Heavy Traffic and Light Traffic Conditions}

Problem one. Determine the performance of your solution in light and heavy traffic.

heavy traffic.: In Model 2, we have analyzed its performance

light traffic: Assuming the vehicle flow rate is 1000 ( $\mathrm{pcu} / \mathrm{h}$ ), the result of vissim simulation is taken as the value of the throughput and accident rate, and the result of model 2 is taken as the cost. The performance of the design is analyzed by using the performance analysis model of Model I and compared with the performance of the charged square under this condition.

$\mathrm{z}(1)=0.3533 * 7+0.2451 * 3+0.2075 * 9-0.1941 * 8=3.4531$

$\mathrm{z}(2)=0.3533 * 5+0.2451 * 3+0.2075 * 9-0.1941 * 5=3.4588$

Results: In the case of heavy traffic, the performance of the design is better than the performance of the toll station has been implemented.

Problem two. How does your solution change as more autonomous (self-driving) vehicles are added to the traffic mix?

The mileage of autonomous (self-driving) vehicles will be far greater than the the mileage of ordinary vehicle. In a situation where the situation is complex, the machine is less capable than the human, so it will increase the accident rate. We can increase the $\mathrm{H}$ and $\mathrm{A}$ to reduce the accident rate and increase throughput.

Problem three. How is your solution affected by the proportions of conventional (human-staffed) tollbooths, exact-change (automated) tollbooths, and electronic toll collection booths (such as electronic toll collection via a transponder in the vehicle)?

Since our design increases the size of the merged area and the signal system is designed in the combined area. Although the accident rate and throughput are reduced, the cost increases. For some financially limited management, it will have to reduce other aspects of toll collection, such as increasing the number of closed toll stations when traffic is low. The passing capacity of the proportions of conventional (human-staffed) tollbooths and exact-change (automated) tollbooths is less than the passing capacity of etc, and the passing capacity of human-staffed is lower than the automatic toll gate. So our design may make the rate of proportions of conventional (human-staffed) tollbooths and exact-change (automated) tollbooths decreased and the rate of etc will increase.

\section{Sensitivity analysis}

Merge mode can not be changed. According to our model I and model II analysis, with the increase of $\mathrm{A}$ and $\mathrm{H}$, the accident prevention effect, congestion evacuation effect will become better, throughput will be large, but the cost will become larger, so the increase of $\mathrm{A}$ and $\mathrm{H}$ should be limited.

When $\mathrm{A}$ is fixed, within a certain limit increase $\mathrm{H}$, although the cost increases, the performance of the consolidation region increases. When $\mathrm{H}$ is fixed, within a certain limit increase $\mathrm{A}$, although the cost increases, the performance of the consolidation region increases. After vissim simulation, the relationship between performance and $\mathrm{A}, \mathrm{H}$ as shown in fig. 17 


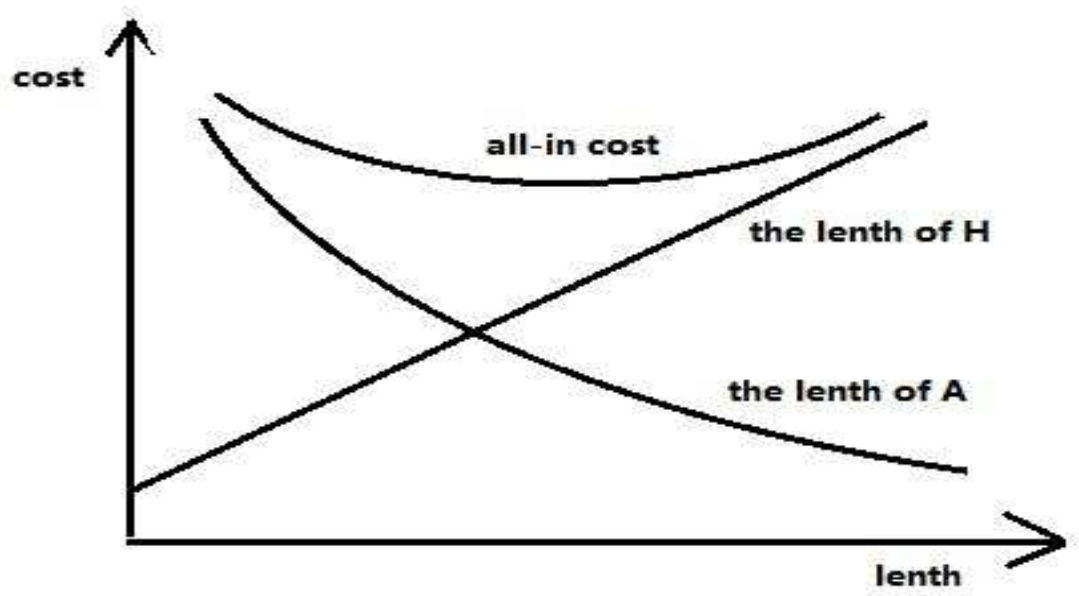

Fig. 17

\section{Advantages and Disadvantages of Models}

Advantages of the model. Our model designs a new merge mode and uses the simulation software to get the data. The conclusion is persuasive.

From the simulation results, the merger model that we designed can effectively improve the performance of the system.

Disadvantages of the Model. We do not take into account the impact of weather, holidays and unexpected events. Our study is based on 6 to 3 traffic patterns. Subjective factors can affect the evaluation of performance.

\section{References}

[1] Li Zhihong, Kong Lingqi, Luo Qiang. Safety evaluation of expressway operation [J]. Chinese Journal of Safety Science, 2008,18 (to): 48-52(In Chinese)

$\mathrm{Hu}$ Jiang Bi. Study on the current situation and development trend of foreign road traffic safety system [J]. Northeast Highway, 2003,26 (3): 121-123

[2] Zhang Chenchen, Wang Yanhui, Jia Limin, congestion control of expressway toll station congestion control strategy, Journal of China Journal of Highway Engineering, July 2013. (In Chinese)

[3] Zhang Min, Chen Hong, Wu Xiaowu, Safety Evaluation Model of Toll Station of Expressway, Chinese Journal of Safety Science, October 2009(In Chinese)

[4] US Department of Transportation official website: www.transportation.gov/

[5] Anastasia D. Spiliopoulou; Ioannis Papamichail; and Markos Papageorgiou Toll Plaza Merging Traffic Control for Throughput Maximization, 10.1061/ ASCE 0733-947X 2010 136:1 67

[6] HUANG Juan-juan, ZHOU Zhou, LIN Xiang-sheng, Vehicle behavior analysis method and system, G08G1 / 01(In Chinese)

[7] Cassidy, M. J., and Rudjanakanoknad, J. 2005. "Increasing the capacityof an isolated merge by metering its on-ramp." Transp. Res., Part B:Methodol., 39, 896-913

[8] Keen, K. G., Schoffield, M. J., and Hay, G. C. 1986. "Ramp metering access control on M6 freeway." Proc., 2nd IEE Int. Conf. on Road Traffic Control, IEE, London.

[9] Kosmatopoulos, E. B., and Papageorgiou, M. 2003. "Stability analysis of the freeway ramp metering control strategy ALINEA." 11th IEEE Mediterranean Conf. on Control and Automation CD-ROM,

Rhodes, Greece

[10] Papageorgiou, M., Hadj-Salem, H., and Blosseville, J. M. 1991. "ALINEA: A local feedback control law for on-ramp metering." Transportation Research Record. 1320, Transportation Research Board, Washington, D.C., 58-64 